University of Wollongong

Research Online

Faculty of Engineering and Information

Faculty of Engineering and Information

Sciences - Papers: Part A

Sciences

$1-1-2014$

Control of conducting polymer actuators without physical feedback: Simulated feedback control approach with particle swarm optimization

Xingcan Xiang

University of Wollongong, xx985@uowmail.edu.au

Rahim Mutlu

University of Wollongong, rm991@uowmail.edu.au

Gursel Alici

University of Wollongong, gursel@uow.edu.au

Weihua Li

University of Wollongong, weihuali@uow.edu.au

Follow this and additional works at: https://ro.uow.edu.au/eispapers

Part of the Engineering Commons, and the Science and Technology Studies Commons

Research Online is the open access institutional repository for the University of Wollongong. For further information contact the UOW Library: research-pubs@uow.edu.au 


\title{
Control of conducting polymer actuators without physical feedback: Simulated feedback control approach with particle swarm optimization
}

\begin{abstract}
Conducting polymer actuators have shown significant potential in articulating micro instruments, manipulation devices, and robotics. However, implementing a feedback control strategy to enhance their positioning ability and accuracy in any application requires a feedback sensor, which is extremely large in size compared to the size of the actuators. Therefore, this paper proposes a new sensorless control scheme without the use of a position feedback sensor. With the help of the system identification technique and particle swarm optimization, the control scheme, which we call the simulated feedback control system, showed a satisfactory command tracking performance for the conducting polymer actuator's step and dynamic displacement responses, especially under a disturbance, without needing a physical feedback loop, but using a simulated feedback loop. The primary contribution of this study is to propose and experimentally evaluate the simulated feedback control scheme for a class of the conducting polymer actuators known as tri-layer polymer actuators, which can operate both in dry and wet media. This control approach can also be extended to other smart actuators or systems, for which the feedback control based on external sensing is impractical.
\end{abstract}

\section{Keywords}

particle, approach, simulated, feedback, conducting, physical, actuators, without, control, optimization, swarm, polymer

\section{Disciplines \\ Engineering | Science and Technology Studies}

\section{Publication Details}

Xiang, X., Mutlu, R., Alici, G. \& Li, W. (2014). Control of conducting polymer actuators without physical feedback: Simulated feedback control approach with particle swarm optimization. Smart Materials and Structures, 23 (3), 035014-1-035014-12. 


\title{
Control of Conducting Polymer Actuators without Physical Feedback: Simulated Feedback Control Approach with Particle Swarm Optimization
}

\author{
Xingcan Xiang ${ }^{1}$, Rahim Mutlu ${ }^{1}$, Gursel Alici ${ }^{1,2}$ and Weihua $\mathbf{L i}^{1}$ \\ ${ }^{1}$ School of Mechanical, Materials and Mechatronic Engineering \\ ${ }^{2}$ ARC Centre of Excellence for Electromaterials Science \\ University of Wollongong, NSW, 2522, Australia \\ E-mail: xx985@uowmail.edu.au
}

\begin{abstract}
Conducting polymer actuators have shown significant potential in articulating micro instruments, manipulation devices, and robotics. However, implementing a feedback control strategy to enhance the positioning ability and accuracy of them in any application requires a feedback sensor, which is extremely large in size compared to the size of the actuators. Therefore, this paper proposes a new sensorless control scheme without the use of a position feedback sensor. With the help of the system identification technique and particle swarm optimization, the control scheme, which we call the simulated feedback control system, showed a satisfactory command tracking performance for the conducting polymer actuator's step and dynamic displacement responses, especially under a disturbance, without needing a physical feedback loop, but using a simulated feedback loop. The primary contribution of this study is to propose and experimentally evaluate the simulated feedback control scheme for a class of the conducting polymer actuators known as tri-layer polymer actuators, which can operate both in dry and wet media. This control approach also can be extended to other smart actuators or systems, for which the feedback control based on external sensing is impractical.
\end{abstract}

Keywords. Actuators, sensorless control, simulated feedback control, intelligent materials, system identification, particle swarm optimization, PID control. 


\section{Introduction}

Electroactive polymer actuators (EAPs) are emerging smart actuators suitable to many cutting-edge applications in robotics and biomedical systems. Inherently conjugated polymers (ICPs), which are also called inherently conducting polymers is one of the main categories of EAPs. The actuators made of ICPs, called conducting polymer actuators (CPAs), are commonly used and show highly favourable characteristics such as minimal electric power consumption, lightweight, biocompatibility, ability to operate in aqueous and non-aqueous media and insensitivity to magnetic fields [1-8]. On the other hand, they possess some undesirable characteristics such as drift/creep, low response speed and high dependency on the fabrication conditions [9-13].

Significant research has been undertaken to improve the control performance of the conducting polymer actuators, and hence enhance their positioning ability and accuracy [10, 14-20]. However, most of the proposed control strategies are based on the traditional feedback control system, in which the displacement output of the CPA need to be acquired from the feedback sensor (laser measurement, for example), which is large in size and weight compared to the actuator.

Moreover, considering the large time constant [2] and unknown system dynamics of CPAs, the existing control strategies still need to be improved for further performance enhancement without adjusting the chemistry of conducting polymers [21-24]. In recent years, some attempts have been made to optimize the synthesis conditions of the CPAs, minimizing the need for accurate mathematical models and feedback control. However, these attempts have not delivered expected outcomes due to conflicting synthesis parameters and their sensitivity to the operating conditions and the size of the actuators, still needing a feedback control system to accurately control the displacement output of these actuators.

In this paper, we present a simple, but effective simulated feedback control system for conducting polymer actuators and other smart actuators. This scheme can control the tip position of CPA without using a displacement feedback sensor, which makes it suitable for the applications in which feedback sensor is impractical. The feedback data is provided through feeding the control input signal to the actuator model, which provides the expected output of the actuator and this output is fedback to the controller for processing and determining the control signal. We have employed a PID (proportional + integral+ derivative) control strategy whose gains are determined using a particle swarm optimization method in order to obtain the best controller parameters and optimize the overall control performance.

The primary contribution of this study is to propose a feedback control strategy which does not require a physical feedback sensor, but still provides the feedback data through the actuator model identified experimentally. We have compared the performance of the proposed control with that of an inversion-based controller, which is an open loop controller not requiring a feedback sensor, and that of a PID controller. We have found that the proposed control strategy is effective enough to enhance the positioning ability of the actuators even under an external disturbance. This study contributes to the previous studies from the point of view of enhancing the positioning ability of these actuators without physically needing feedback sensors.

First, we introduce the research background in Section 2. Then we present the experimental setup and the method for driving the conducting polymer actuators in Section 3. The model for actuator displacement was identified and the feedback control strategy is described and applied on the CPA control system in Section 4. In Section 5, we compare the proposed control strategy with the inversion-based feedforward control. Finally, concluding remarks are provided in Section 6. 


\section{Background}

Polypyrrole (PPy) is one of the most commonly used ICPs for actuation purposes. This smart material has been widely developed for actuator applications because they can produce larger strains and low or medium stresses at low voltages, compared to other actuator materials [10].

A PPy polymer actuator consists of three main layers, shown in figure 1. The middle layer is poly(vinylidene fluoride) (Immobilon-P, Millipore) (PVDF) which is also the electrolyte reservoir. The outside layers are PPy layers. Between the PVDF layer and each of the PPy layers, a very thin gold layer (clusters of gold particles with enough porosity in between) is introduced to improve the conductivity.

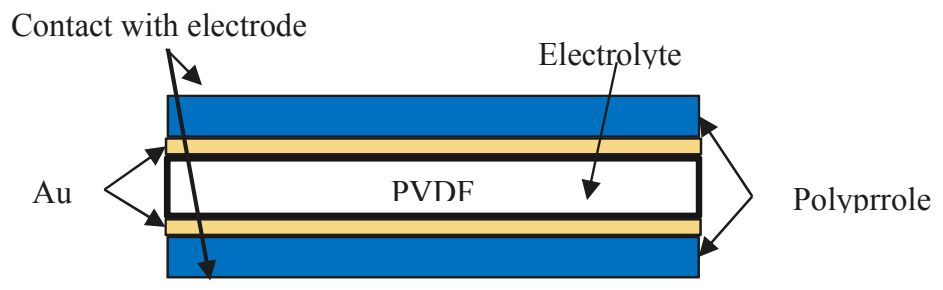

Figure 1: The structure of a PPy actuator, whose one-end is fixed to operate like a cantilever beam. While its one PPy layer is expanding, the other contracts to generate a strain difference between both layers like a bimetal in order to output a significant deflection at its tip point.

The PPy layers are used as an electrode. The one contacted with the anode will be oxidized and this process will result in the swell of the PPy layers. By contrast, the contraction of the PPy polymer will result from the reduction process at the cathode (figure 2). As a result, the trilayer actuator will bend and the displacement of the actuator tip can be controlled by adjusting the voltage applied at the fixed end.

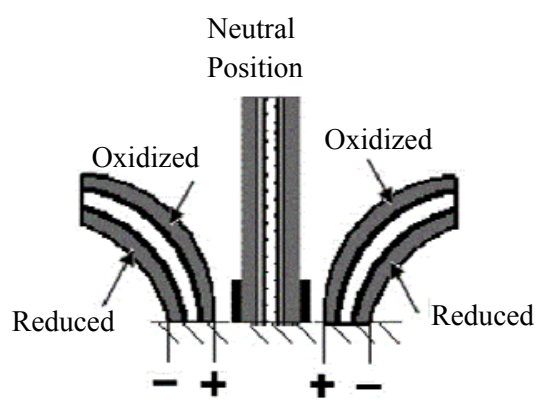

Figure 2: The bending directions of the tri-layer PPy actuator [5].

\section{The Experimental Setup}

\subsection{The Fabrication of Trilayer Conducting Polymer Actuators}

PPy conducting polymer actuator (CPA) has a laminated trilayer structure. It is composed of a goldcoated PVDF substrate and polypyrrole layers. The gold coating on the PVDF membrane is finished by a sputter-coating machine. To polymerize the PPy layers outside the PVDF substrate, we used electrochemical polymerization, using the setup in figure 3. A glass cell was constructed from plate glasses and engineering silicone was used as the container of the polymerization. The gold coated PVDF film and stainless steel mesh were placed in the container full of polymerization solution, $0.1 \mathrm{M}$ $\mathrm{Li}^{+}{ }^{+} \mathrm{TFSI}^{-}$in propylene carbonate (PC). The stainless steel meshes were used as the counter electrode and connected to the reference electrode. The PVDF film was connected to the working electrode. It 
takes 12 hours in the freezer for completing the polymerization process to obtain a substrate with the PPy layer thickness of 30 um.

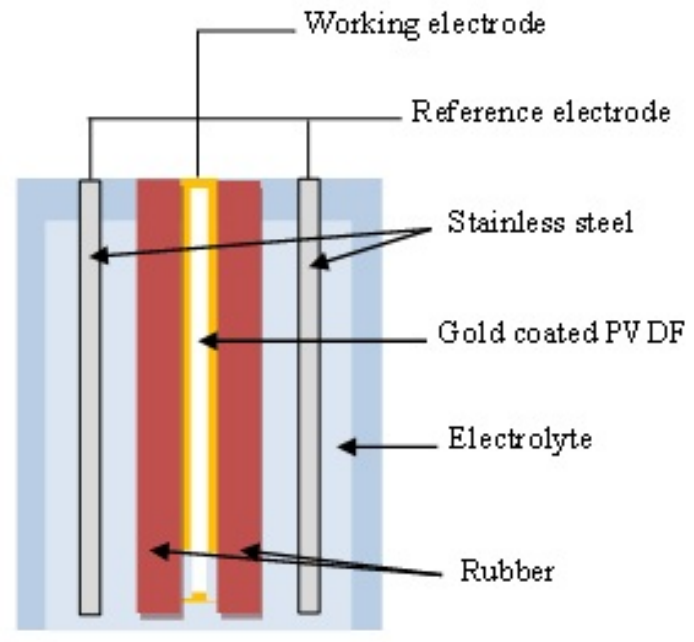

Figure 3: The schematic of the glass polymerization cell, with the growth solution, gold coated PVDF, rubber and stainless steel mesh.

After polymerization, a polymer actuator with an arbitrary shape and size can be cut from the actuator substrate by a sharp scalpel or laser. The $15.3 \mathrm{~mm}$-long, $3.3 \mathrm{~mm}$-wide and 0.17 -mm-thick sample was trimmed from the polymer sheet and used in our experiments.

\subsection{Actuator Driving and Displacement Measuring System}

A custom-built experimental setup is used to provide measure and record all the input and output signals of the CPA. Figure 4 depicts the arrangement of this setup. The actuator input signal was first generated by the control computer, then interfaced through a data acquisition board (NI 6251 and SCB-68) and amplified using a potentiostat (e-DAQ). The potentiostat has two electrodes which are connected to two sides of the actuator. A noncontact laser displacement sensor was employed to measure the displacement of the actuator tip. Figure 5 shows the photograph of the experimental setup.

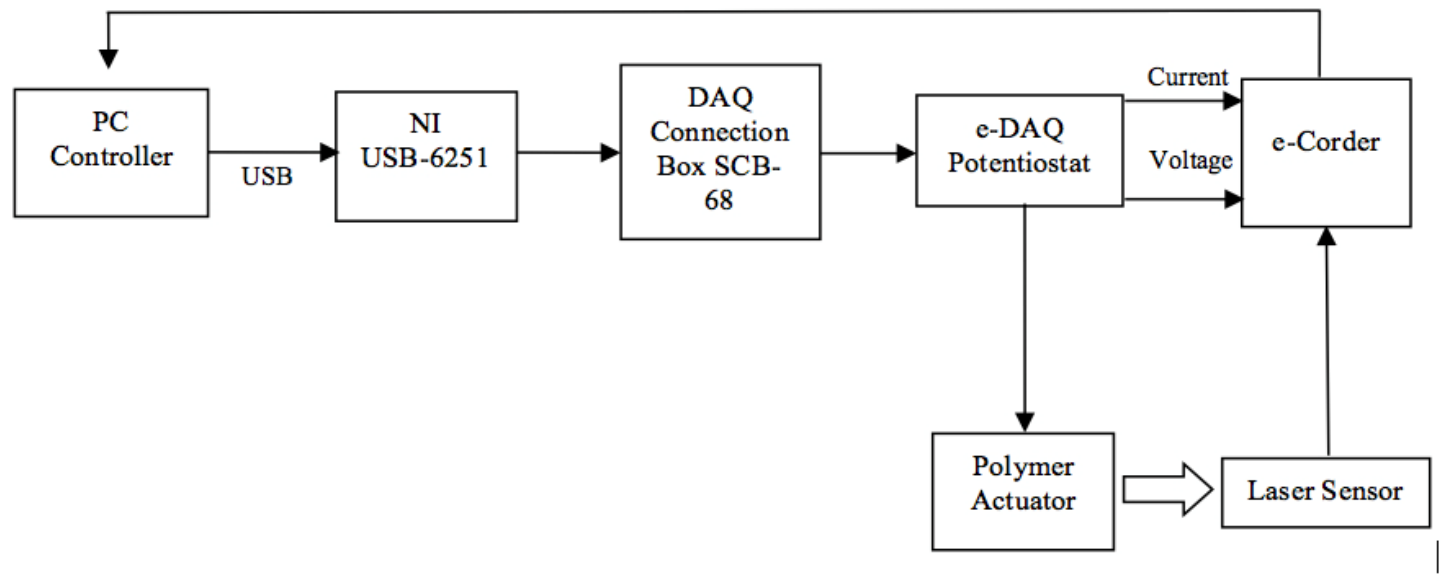

Figure 4: Schematic representation of the experimental setup. 


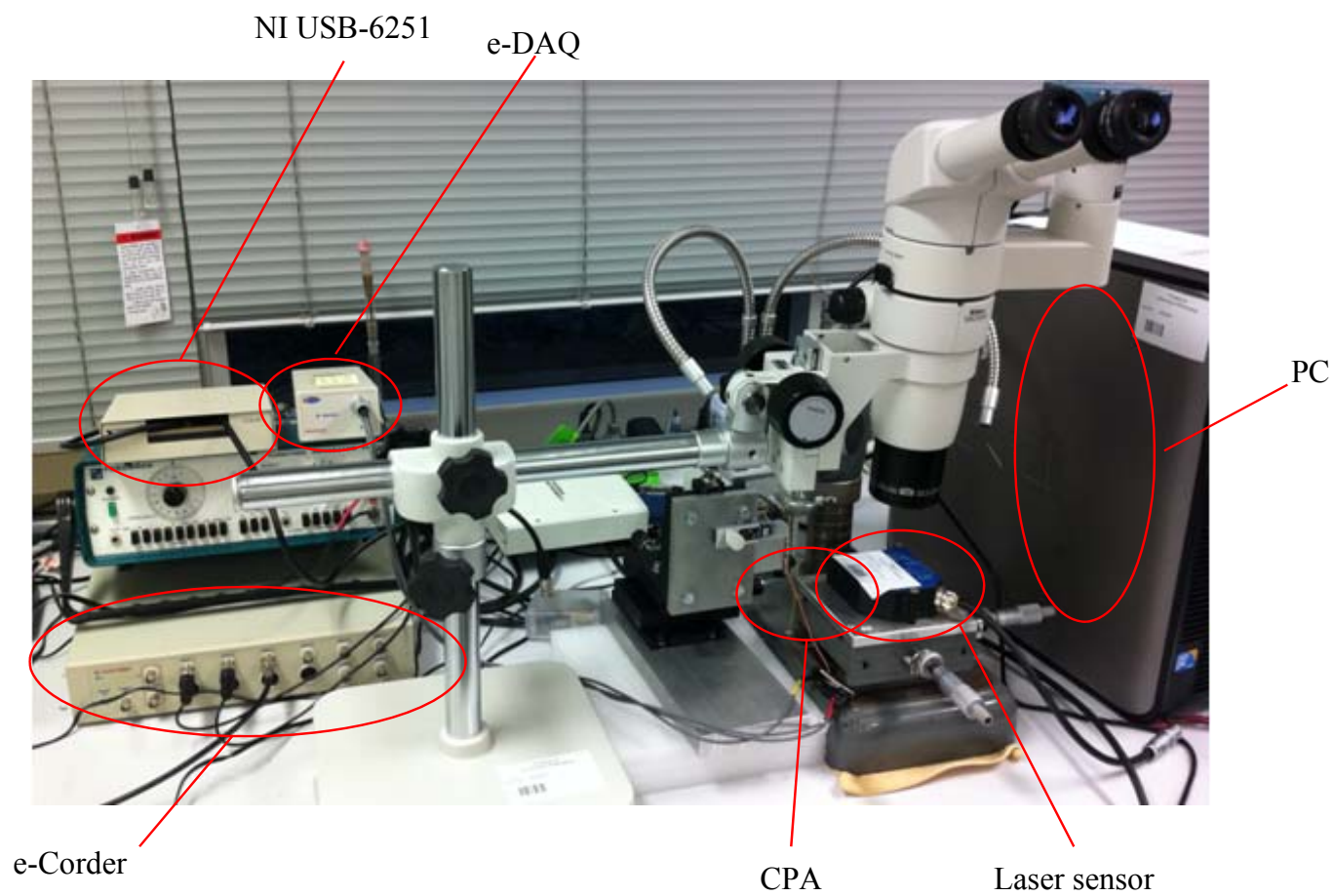

Figure 5: Photograph of the experimental setup for CPA control system.

\section{Inversion-based Feedforward Control Strategy}

An Inversion-based feedforward control (IFC) strategy was previously proposed and evaluated for the tri-layer conducting polymer actuators considered in this study [9]. This strategy aims to improve the trilayer actuator position control performance without the use of a feedback sensor.

For a given system, $Y(s)=U(s) G(s)$, where $U(s)$ is the control input and $Y(s)$ is the actuator output, the input signal would achieve a desired output when the controller output is calculated from $U_{c}(s)=Y_{d}(s) G^{-1}(s)$ provided that the inverse plant $G^{-1}(s)$ will compensate for the actuator dynamics. The schematic diagram of the IFC strategy is shown in figure 6.

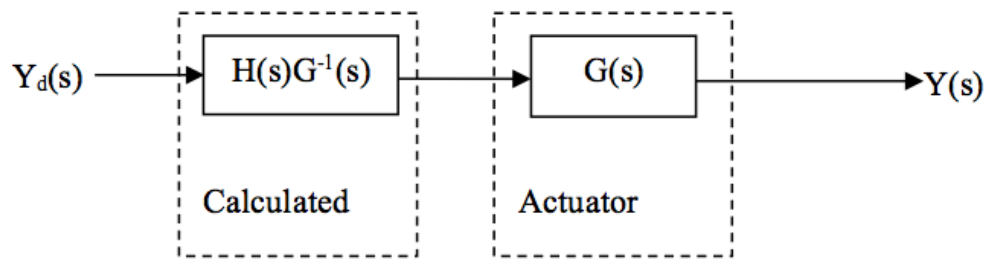

Figure 6: Schematic diagram of the inversion-based feedforward control system.

After identifying the actuator model, $G^{-1}(s)$ can be obtained by direct inversion, provided that all poles and zeros are on the left-hand side of the s-plane. A Bessel (low-pass) filter ( $H(s)$ shown in figure 6) had to be used as the inverted plant $G^{-1}(s)$ has a greater number of zeros than poles and it is unrealizable. 


\section{Simulated Feedback Control Approach}

\subsection{Principle of Operation}

As shown in figure 7, the actuator model was estimated as the "Identified model" in the feedback control loop. The feedback control was established in the simulated environment and the parameters of the controller can be tuned in the software carefully according to this identified model to obtain the high performance simulation output.

We sent the controller's output in the simulated feedback system to the real plant, i.e., actuator. Theoretically, the real output should be identical with the simulation output as the "Identified model" and "Real plant" are expected to be equivalent. This follows that we can implement a feedback control system without physically using a feedback sensor and feedback loop. We call the proposed control scheme the simulated feedback control (SFC) since the feedback loop is established in the simulated environment rather than in the real world. However, it must be noted that the control performance depends on the accuracy of the identified model. It is important to estimate the plant model as accurate as possible.

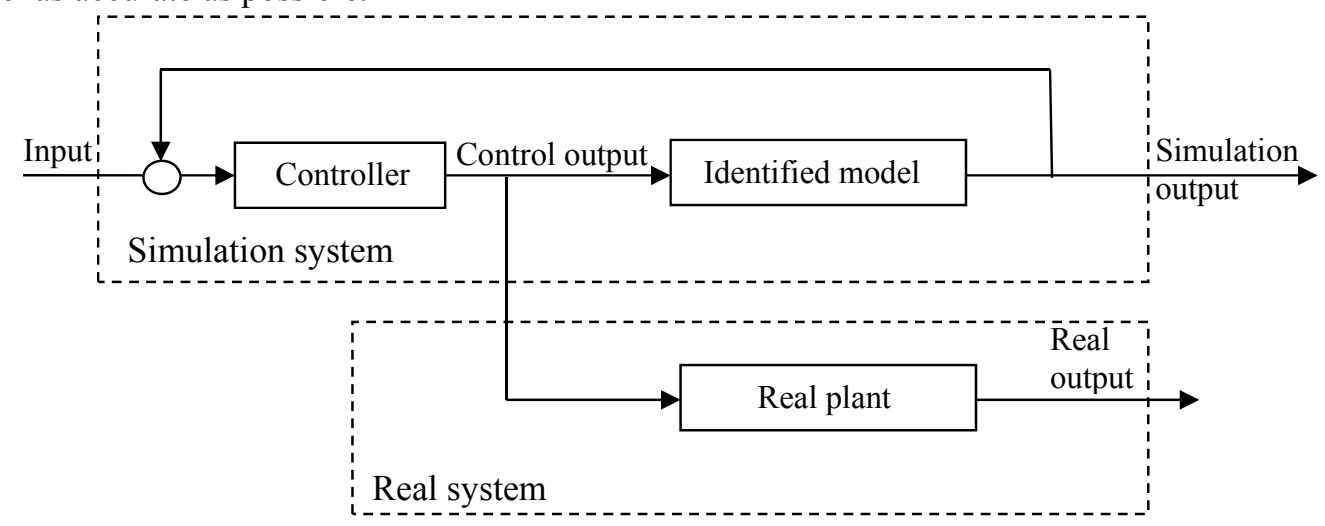

Figure 7: The schematic of the simulated feedback control system.

\subsection{System Identification}

The linear system can be expressed as a transfer function:

$$
G(s)=\frac{y(s)}{u(s)}=\frac{b_{0} s^{m}+b_{1} s^{-1}+\ldots+b_{m-1} s+b_{m}}{a_{0} s^{n}+a_{1} s^{n-1}+\ldots+a_{n-1} s+a_{n}}
$$

This transfer function is given by

$$
a_{0} s^{n} y(s)+a_{1} s^{n-1} y(s)+\ldots+a_{n-1} s y(s)+a_{n} y(s)=b_{0} s^{m} u(s)+b_{1} s^{-1} u(s)+\ldots+b_{m-1} s u(s)+b_{m} u(s)
$$

This equation can be written as a differential equation:

$$
\begin{aligned}
& a_{0} \frac{\mathrm{d}^{n}}{\mathrm{~d} t^{n}} y(t)+a_{1} \frac{\mathrm{d}^{n-1}}{\mathrm{~d} t^{n-1}} y(t)+\cdots+a_{n-1} \frac{\mathrm{d}}{\mathrm{d} t} y(t)+a_{n} y(t) \\
& =b_{0} \frac{\mathrm{d}^{m}}{\mathrm{~d} t^{m}} u(t)+b_{1} \frac{\mathrm{d}^{m-1}}{\mathrm{~d} t^{m-1}} u(t)+\cdots+b_{m-1} \frac{\mathrm{d}}{\mathrm{d} t} u(t)+b_{m} u(t)
\end{aligned}
$$

So we have

$$
y(t)=-a_{0} \frac{\mathrm{d}^{n}}{\mathrm{~d} t^{n}} y(t)-a_{1} \frac{\mathrm{d}^{n-1}}{\mathrm{~d} t^{n-1}} y(t)-\cdots-a_{n-1} \frac{\mathrm{d}}{\mathrm{d} t} y(t)-\left(a_{n}-1\right) y(t)
$$




$$
+b_{0} \frac{\mathrm{d}^{m}}{\mathrm{~d} t^{m}} u(t)+b_{1} \frac{\mathrm{d}^{m-1}}{\mathrm{~d} t^{m-1}} u(t)+\cdots+b_{m-1} \frac{\mathrm{d}}{\mathrm{d} t} u(t)+b_{m} u(t)
$$

Now

$$
y=F p
$$

Where

$F=\left[y^{n}, y^{n-1}, \ldots, y^{\prime}, y, u^{m}, u^{m-1}, \ldots, u^{\prime}, u\right]$,

$p=\left[-a_{0},-a_{1}, \ldots,-a_{n-1},-\left(a_{n}-1\right), b_{0}, b_{1}, \ldots, b_{m-1}, b_{m}\right]^{T}$.

If the matrix $F^{T} F$ is non-singular, the parameter estimated by minimising the least squares index is given by

$$
\hat{p}=\left(F^{T} F\right)^{-1} F^{T} y
$$

Considering the relationship between the input and output to be a "black box", the system order and delays should be fixed first. We considered a range of orders, from a second-order to a sixth-order, and found the second order model fitted to the experimental data well. The fit rate between the model described behavior and the real CPA behavior is more than $95 \%$, as shown in figure 8 .

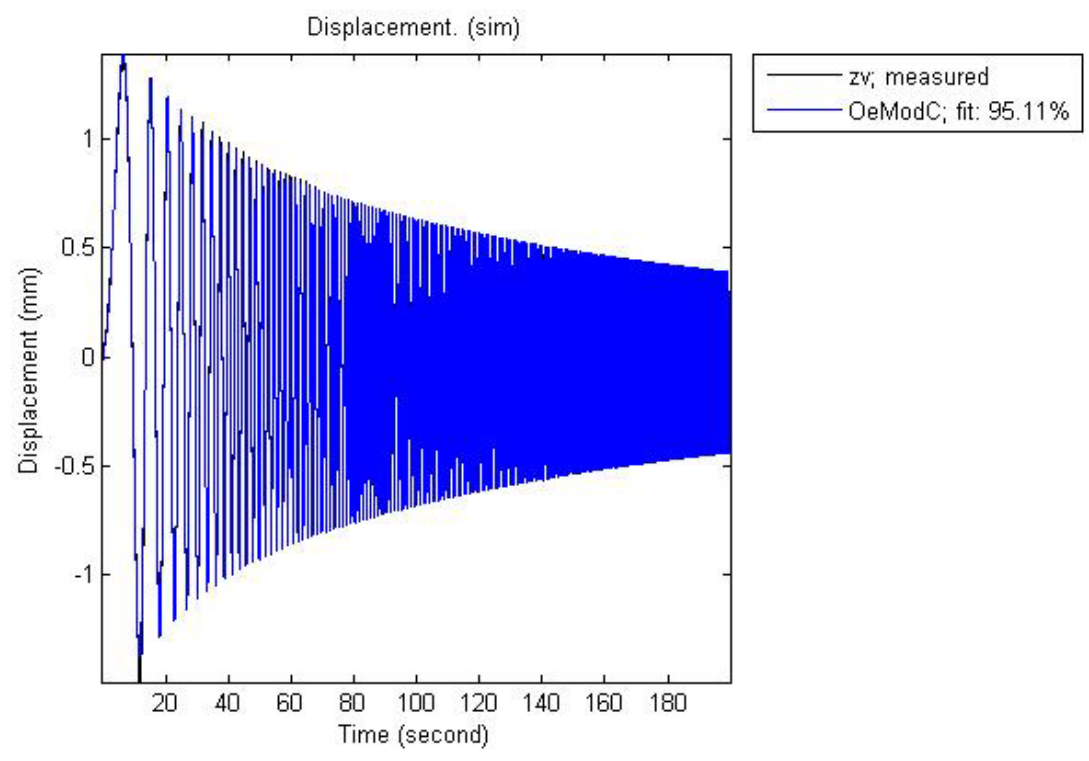

Figure 8: The fit rate of the identified model. The output of the estimated model (blue line) is very close to the measured experimental data (black line) and they are almost overlapped each other.

In the identification process, we have taken the time delay into the mathematical model of the conducting polymer actuator to improve the model accuracy when implementing the simulated control system. We determined the time delay of $18 \mathrm{~ms}$. The resulting transfer function including the time delay is given by

$$
G(s)=e^{-0.018 s} \frac{11.72 s+33.05}{s^{2}+8.68 s+11.4}
$$


With reference to [9], we have not considered the time delay when implementing the inversion-based feedforward control system.

\subsection{Development of the Controller}

The identified model was used to generate the control signal in the proposed simulated feedback control system. To improve the model accuracy, the time delay element is retained but a fourth-order transfer function (Pade approximation) is chosen:

$$
G_{d}=\frac{s^{4}-1.1111 \times 10^{3} s^{3}+5.5556 \times 10^{5} s^{2}-1.4403 \times 10^{8} s+1.6004 \times 10^{10}}{s^{4}+1.1111 \times 10^{3} s^{3}+5.5556 \times 10^{5} s^{2}+1.4403 \times 10^{8} s+1.6004 \times 10^{10}}
$$

In the simulated control approach, the PID controller is used due to its very good control performance and simple structure. However, it is not straightforward to find the best PID gains. Therefore, we employ a particle swarm optimization algorithm to determine the gains.

\subsection{Particle Swarm Optimization for Tuning PID Gains}

Particle swarm optimization is a kind of evolutionary computation technique to solve a nonlinear optimization problem. In this algorithm, a number of particles are placed in a multidimensional search space. Each of them flies in the space with a specific velocity. Every particle's velocity is dynamically adjusted according to its own flying experience and overall swarm flying experience. Eventually, the swarm, like a flock of birds catching food, would move close to the optimized point of the problem $[26,27]$.

The $j$ th particle which is in the g-dimension space can be represented as $x_{j}=\left(x_{j, 1}, x_{j, 2}, \ldots, x_{j, g}\right)$, and the best previous position of particle $j$ can be represented as pbest $_{j}=\left(\right.$ pbest $_{j, 1}$, pbest $_{j, 2}, \ldots$, pbest $\left._{j, g}\right)$. The velocity of the particle $j$ can be represented as $v_{j}=\left(v_{j, 1}, v_{j, 2}, \ldots, v_{j, g}\right)$. Then (9) and (10) can be used to calculate the particle's new position and velocity [28].

$$
\begin{gathered}
v_{\mathrm{j}, \mathrm{g}}^{(t+1)}=w \cdot v_{\mathrm{j}, \mathrm{g}}^{(t)}+c_{1} * \operatorname{rand}_{1}() *\left(\text { pbest }_{\mathrm{j}, \mathrm{g}}-x_{\mathrm{j}, \mathrm{g}}^{(t)}\right) \\
+c_{2} * \operatorname{rand}_{2}() *\left(\text { gbest }_{\mathrm{j}, \mathrm{g}}-x_{\mathrm{j}, \mathrm{g}}^{(t)}\right) \\
x_{\mathrm{j}, \mathrm{g}}^{(t+1)}=x_{\mathrm{j}, \mathrm{g}}^{(t)}+v_{\mathrm{j}, \mathrm{g}}^{(t+1)}
\end{gathered}
$$

$\mathrm{j}=1,2, \ldots, \mathrm{n}$

$\mathrm{g}=1,2, \ldots, \mathrm{m}$

where

$\mathrm{n}$ is the number of particles in the swarm group;

$\mathrm{m}$ is the number of dimensions of a particle;

$t$ is the pointer of the iterations;

$v_{\mathrm{j}, \mathrm{g}}^{(t)}$ is the current velocity of $j$ particle at t iteration;

$x_{\mathrm{j}, \mathrm{g}}^{(t)}$ is the current position of $j$ particle at $\mathrm{t}$ iteration; 
$c_{1}$ and $c_{2}$ are acceleration constant which determine the weights of velocity point to pbest and gbest.

$\operatorname{rand}_{1}()$ and $\operatorname{rand}_{2}()$ are random numbers between 0 and1;

$w$ is the inertia weight factor.

To find the best results, we vary $\mathrm{W}$ to speed up the convergence of the PSO algorithm. $W$ is relatively large (which would help particles move quickly) at the beginning of the optimization process and decreases along with the iteration to make the particles come close to the target position. The numerical value of $W$ is:

$$
W=w_{\max }-\left(w_{\max }-w_{\min }\right) \cdot \frac{\text { iteration }_{\text {current }}}{\text { iteration }_{\max }}
$$

For the PID controller design, the control parameters, the proportional, integral and derivative gains, can be deemed as the three dimensions of one particle. A number of particles in the problem space are looking for their best position, which means the best gains. The criterion of the best position usually comes from some system response index in time domain or frequency domain. We employ a performance criterion $W(K)$, proposed by Zwe-Lee Gaing [28], in our algorithm. Because this criterion can measure the performance comprehensively in the time domain and it can also be adjusted easily according to the design requirement. The $W(K)$ is formulated as

$$
W(K)=\left(1-e^{-\beta}\right) \cdot\left(M_{p}+E_{s S}\right)+e^{-\beta} \cdot\left(t_{s}-t_{r}\right)
$$

These performance criteria in the time domain include the overshoot $M_{p}$, rise time $t_{r}$, settling time $t_{s}$, and steady-state error $E_{s s}$. We can adjust the value of $\beta$ to change the weighting of the control performance parameters in this criterion function.

On the other hand, we also consider the root mean square (RMS) of the response curve as the performance criteria because it is possible to get an optimized response curve measured by the overall error. The RMS performance criterion is defined by:

$$
W^{\prime}(K)=R M S=\sqrt{\frac{1}{n} \sum_{i=1}^{n}\left(Y r_{i}-Y d_{i}\right)^{2}}
$$

where $n$ is the total number of data points, $Y_{r}$ is the real output, and $Y_{d}$ is the desired output.

In general, it only takes 20 to 60 seconds to find the optimized PID gains for the controller. The unstable PID gains are automatically eliminated during the optimization. For the experimentally identified model, PID gains of $k p=1.881, k i=1.9008, k d=0$ are obtained. The resulting optimized response under these gains are shown in figure 9. 


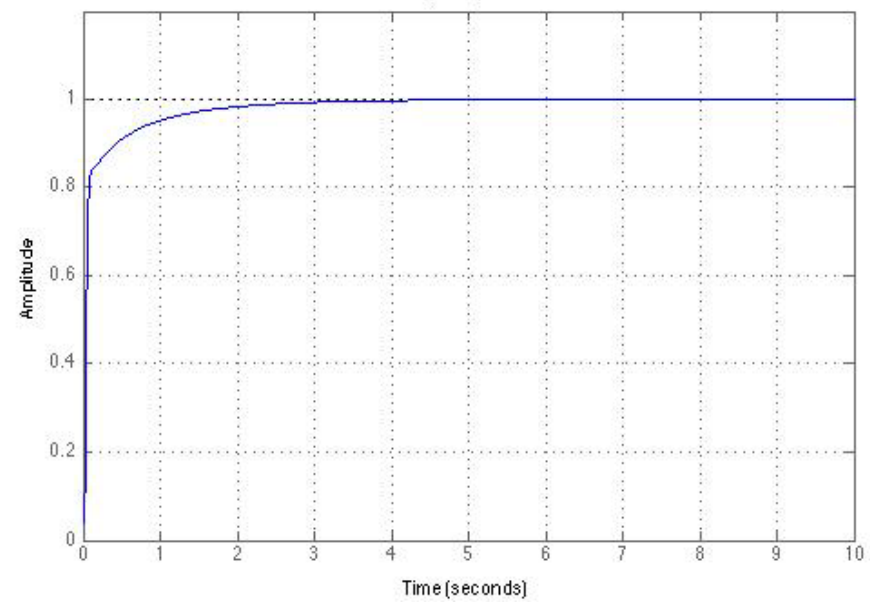

Figure 9: The step response under the PID control optimized by the PSO algorithm.

\subsection{The Step Response}

The control signal calculated by the controller and the resulting experimental displacement response of the CPA under the IFC and SFC schemes are presented in figure 10 and figure 11, respectively.
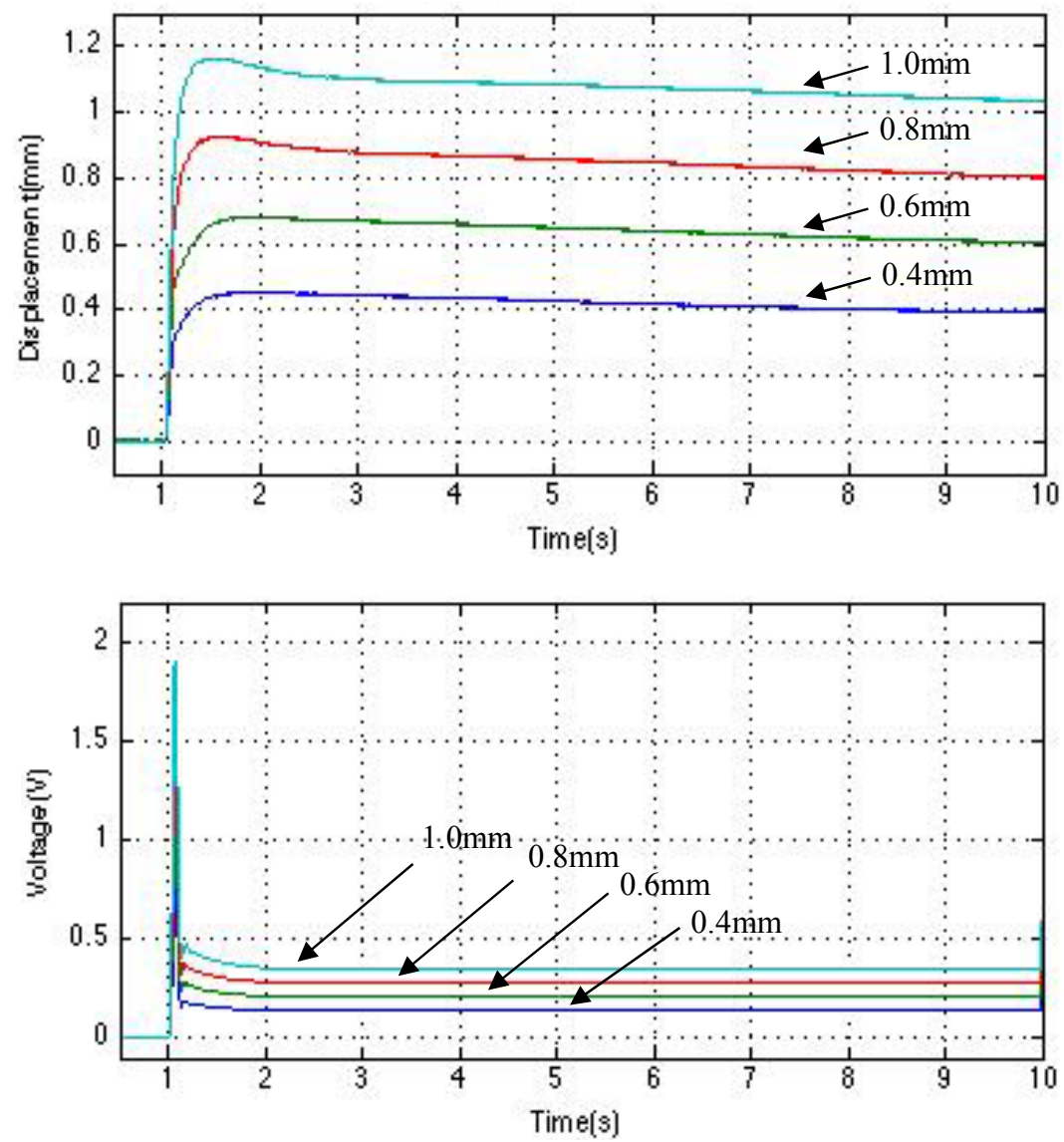

Figure 10: Experimental displacement step responses and control voltage for the CPA under IFC. 

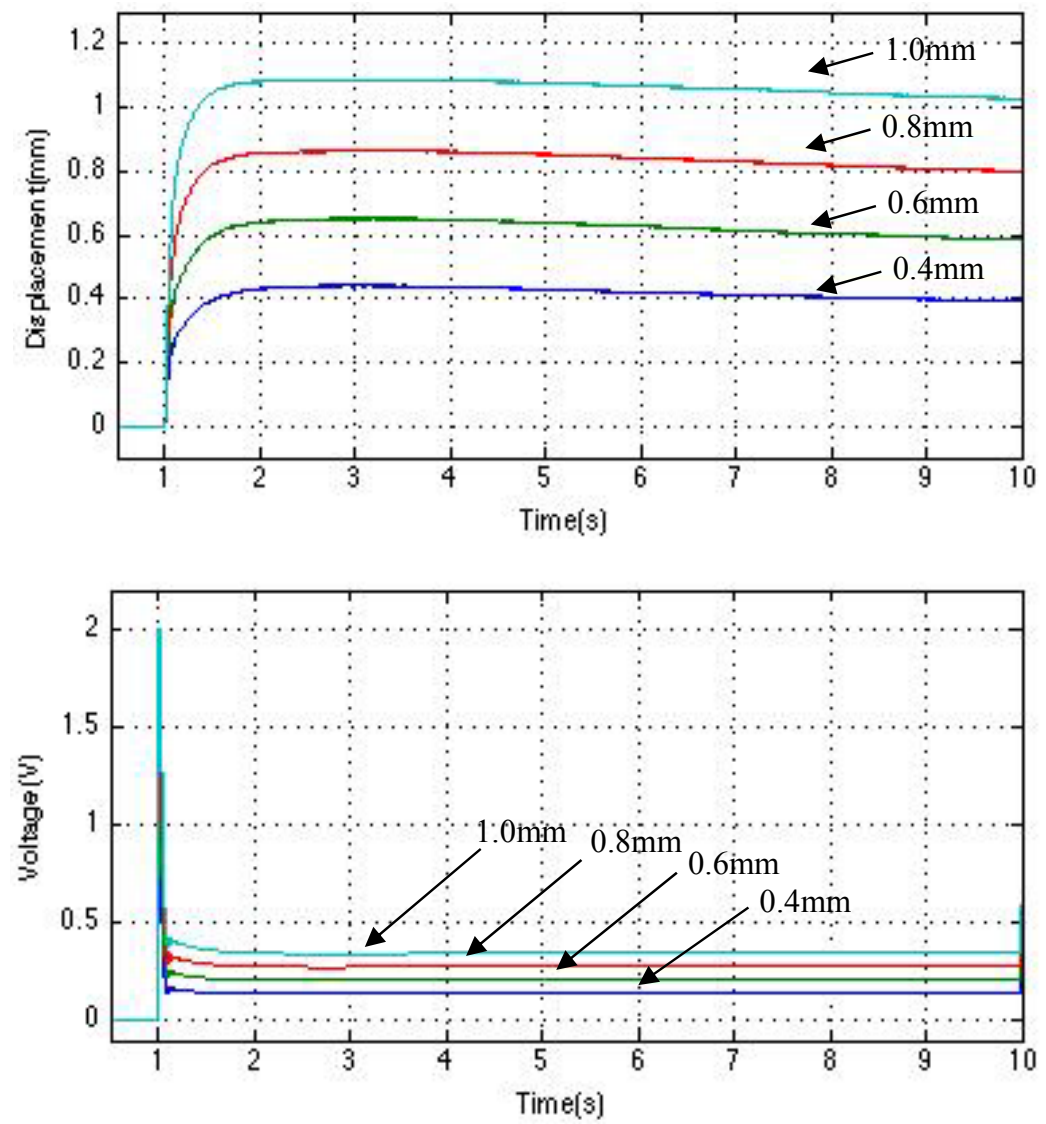

Figure 11: Experimental displacement of step responses and control voltage for the CPA under SFC.

The inversion-based control step responses have been presented with the simulated feedback control step response in figure 12. The displacement response characteristic including overshoot, rise time, settling time, steady state error are depicted in table 1 and table 2 . The rise time for SFC is longer than the IFC rise time. However, in most cases, the settling time for SFC is shorter than that of IFC. The same result was observed for the steady state error. In most situations, the tracking error of SFC is smaller than that of the IFC.

The RMS calculated for these two control strategies is different. The SFC results in a better RMS value in every step response, which indicates a better command tracking ability.

In summary, the results presented show a fast and accurate response under SFC, considering that the rise time of SFC is still very short and acceptable. 


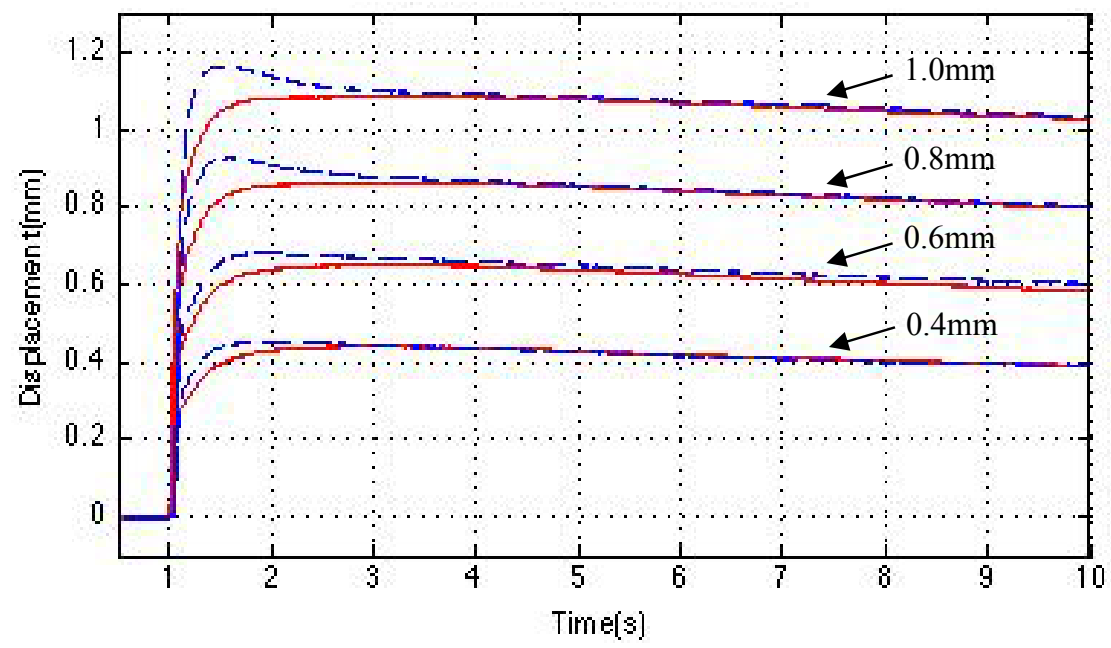

Figure 12: The comparison of the experimental step displacement responses for the CPA under different controller. The red solid line is under SFC controller and the blue dotted line is under IFC controller

Table 1. The transient response characteristics of the CPA under IFC.

\begin{tabular}{cccccc}
\hline $\begin{array}{c}\text { Input } \\
\text { displacement } \\
(\mathrm{mm})\end{array}$ & Overshoot $(\%)$ & Rise time $(\mathrm{s})$ & $\begin{array}{c}\text { Settling time } \\
(\mathrm{s})\end{array}$ & $\begin{array}{c}\text { Steady state } \\
\text { error }(\mathrm{mm})\end{array}$ & RMS \\
\hline 0.4 & $\begin{array}{c}13.3 \% \\
(0.053 \mathrm{~mm}) \\
13.7 \%\end{array}$ & 0.13 & 5.68 & -0.009 & 0.044 \\
0.6 & $\begin{array}{c}(0.082 \mathrm{~mm}) \\
15.9 \%\end{array}$ & 0.16 & 6.91 & 0.003 & 0.068 \\
0.8 & $\begin{array}{c}(0.127 \mathrm{~mm}) \\
16.5 \%\end{array}$ & 0.10 & 6.61 & 0.003 & 0.090 \\
1.0 & $(0.165 \mathrm{~mm})$ & 0.10 & 8.42 & 0.034 & 0.118 \\
\hline
\end{tabular}

Table 2. The transient response characteristics of the CPA under SFC.

\begin{tabular}{cccccc}
\hline $\begin{array}{c}\text { Input } \\
\text { displacement } \\
(\mathrm{mm})\end{array}$ & $\begin{array}{c}\text { Overshoot } \\
(\mathrm{mm})\end{array}$ & Rise time $(\mathrm{s})$ & $\begin{array}{c}\text { Settling time } \\
(\mathrm{s})\end{array}$ & $\begin{array}{c}\text { Steady state } \\
\text { error }(\mathrm{mm})\end{array}$ & RMS \\
\hline 0.4 & $\begin{array}{c}10.5 \% \\
(0.042 \mathrm{~mm}) \\
0.8 \%\end{array}$ & 0.30 & 6.07 & -0.008 & 0.037 \\
0.6 & $\begin{array}{c}8.8 \% \\
\left(\begin{array}{l}0.053 \mathrm{~mm}) \\
8.2 \%\end{array}\right.\end{array}$ & 0.26 & 5.79 & -0.016 & 0.051 \\
0.8 & $\begin{array}{c}(0.065 \mathrm{~mm}) \\
8.6 \%\end{array}$ & 0.23 & 6.21 & 0 & 0.067 \\
1.0 & $(0.086 \mathrm{~mm})$ & 0.22 & 7.80 & 0.027 & 0.089
\end{tabular}


5.6 Step Response with impulse disturbance

A disturbance was applied to test the robustness of the control strategies under disturbances, as shown in figure 13 . We introduced a $0.5 \mathrm{~V}$ impulse disturbance at the time of $10 \mathrm{~s}$. Figure 14 shows the CPA displacement response after impulse disturbance have been introduced.

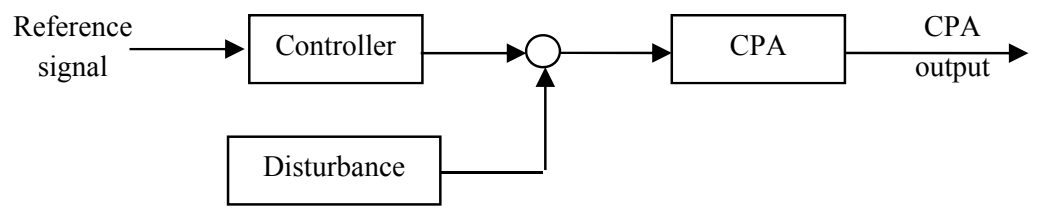

Figure 13: The schematic of control system with disturbance

The results in figure 14 show that the IFC controller is not able to output an adjustment signal when the disturbance is applied. However, due to the feedback structure of the SFC strategy, the SFC controller made a prompt response to the impulse disturbance and adjusted the displacement output of CPA immediately. The RMS values under different step inputs are presented in table 3 to show the performance of these two control strategies. The response curves of SFC have much less RMS values than that of IFC in all input circumstances, which show its superior performance under an external disturbance.
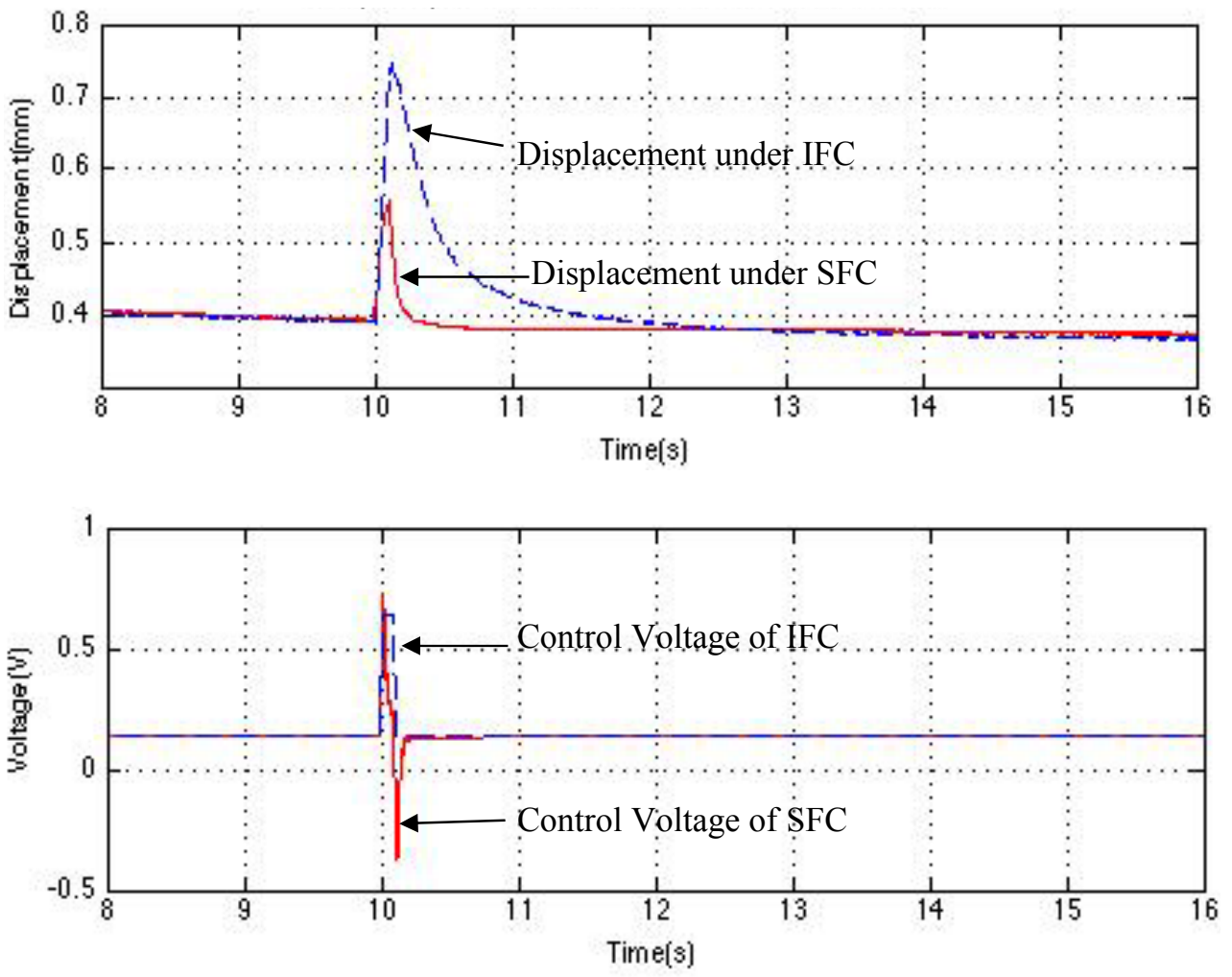

Figure 14: The experimental step response of CPA and its control voltage under IFC and SFC with impulse disturbance

We also measured the maximum deviation from the output under the disturbance and the time needed for the output to recover from the disturbance. This result also shows SFC's better performance, as presented in table 3 . 
Table 3. The performance of the CPA's step response under IFC and SFC with impulse disturbance measured by RMS.

\begin{tabular}{ccccccc}
\hline & \multicolumn{3}{c}{ Inversion-based control } & \multicolumn{3}{c}{ Simulated feedback control } \\
\hline $\begin{array}{c}\text { Input } \\
\text { displacement } \\
(\mathrm{mm})\end{array}$ & $\begin{array}{c}\text { Max } \\
\text { deviation } \\
(\mathrm{mm})\end{array}$ & $\begin{array}{c}\text { Recovering } \\
\text { time (s) }\end{array}$ & RMS & $\begin{array}{c}\text { Max } \\
\text { deviation } \\
(\mathrm{mm})\end{array}$ & $\begin{array}{c}\text { Recovering } \\
\text { time (s) }\end{array}$ & RMS \\
\hline 0.4 & 0.346 & 1.04 & 0.060 & 0.156 & 0.18 & 0.024 \\
0.6 & 0.368 & 1.05 & 0.064 & 0.167 & 0.17 & 0.036 \\
0.8 & 0.380 & 0.93 & 0.068 & 0.184 & 0.17 & 0.030 \\
1.0 & 0.390 & 1.20 & 0.075 & 0.217 & 0.20 & 0.031
\end{tabular}

5.7 Step Response with white noise disturbance

In a practical application, the white noise disturbance is more common than the impulse disturbance. To identify the displacement response with white noise under IFC and SFC controllers, the experimental results were obtained and shown in figure 15.

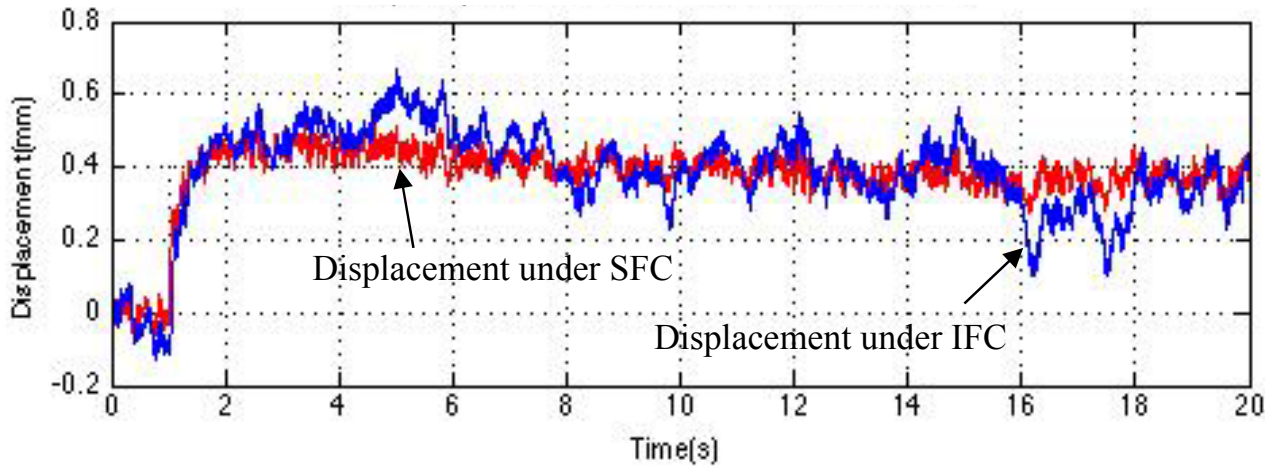

Figure 15: The experimental step response of CPA and its control voltage under IFC and SFC with white noise disturbance.

We have also calculated the RMS value under different step inputs + white noise and presented in table 4. Similar to the response under the impulse disturbance, the IFC controller was unable to response to the disturbance. As the result, the SFC controller shows a better performance.

Table 4. The performance of the CPA's step response under IFC and SFC with white noise disturbance measured by RMS.

\begin{tabular}{ccc}
\hline $\begin{array}{c}\text { Input displacement } \\
(\mathrm{mm})\end{array}$ & $\begin{array}{c}\text { RMS under inversion- } \\
\text { based control }\end{array}$ & $\begin{array}{c}\text { RMS under } \\
\text { simulated feedback } \\
\text { control }\end{array}$ \\
0.4 & 0.098 & 0.046 \\
0.6 & 0.115 & 0.059 \\
0.8 & 0.126 & 0.069 \\
1.0 & 0.144 & 0.085 \\
\hline
\end{tabular}




\subsection{Dynamic Response}

The dynamic response of the CPA was measured under a sinusoidal signal, $F(t)=0.8 \sin (0.1 \pi t)+0.2 \sin$ $(\pi t)$ for the same actuator sample. The RMS of the displacement response under IFC and SFC was derived from the experimental data and used to quantify the overall error of the output displacement. The results in table 5 and figure 16 show the RMS improvement under the SFC, compared to the IFC.
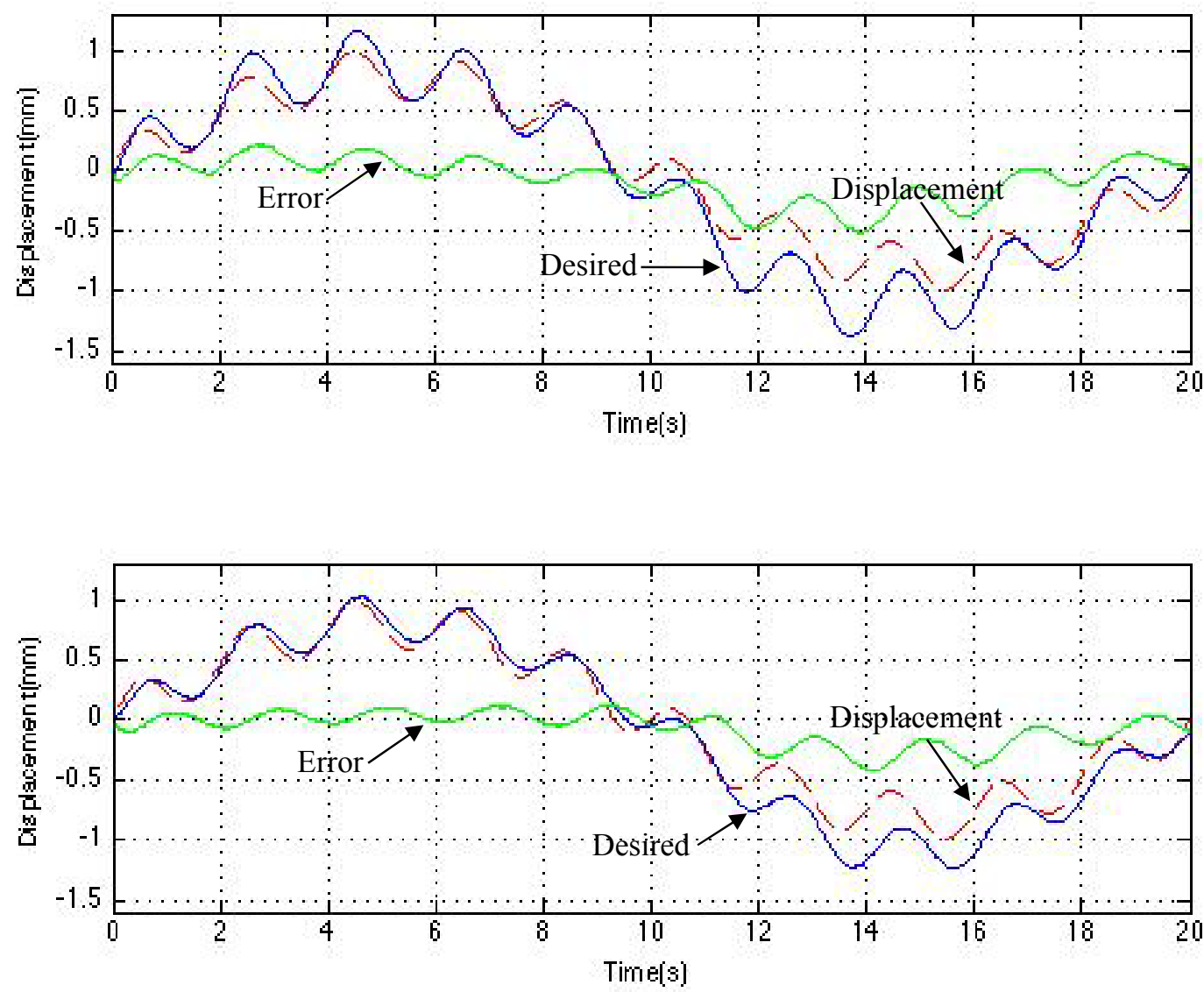

Figure 16: The experimental dynamic response of CPA under IFC and SFC control strategies.

Table 5. The comparison of the dynamic response characteristics of the IFC and SFC strategies.

\begin{tabular}{ccc}
\hline Input & $\begin{array}{c}\text { RMS under inversion-based } \\
\text { control }\end{array}$ & $\begin{array}{c}\text { RMS under simulated feedback } \\
\text { control }\end{array}$ \\
\hline$f(t)=0.8 \sin (0.1 \pi \mathrm{t})+0.2 \sin (\pi \mathrm{t})$ & 0.195 & 0.154 \\
\hline
\end{tabular}

5.9 Dynamic Response under impulse disturbance

As shown in figures 17 and 18 and table 6, the IFC shows a larger error when a disturbance was applied (RMS=0.213). In contrast, the SFC controller makes the output displacement follow the input signal much better, resulting in the RMS of 0.134 . 

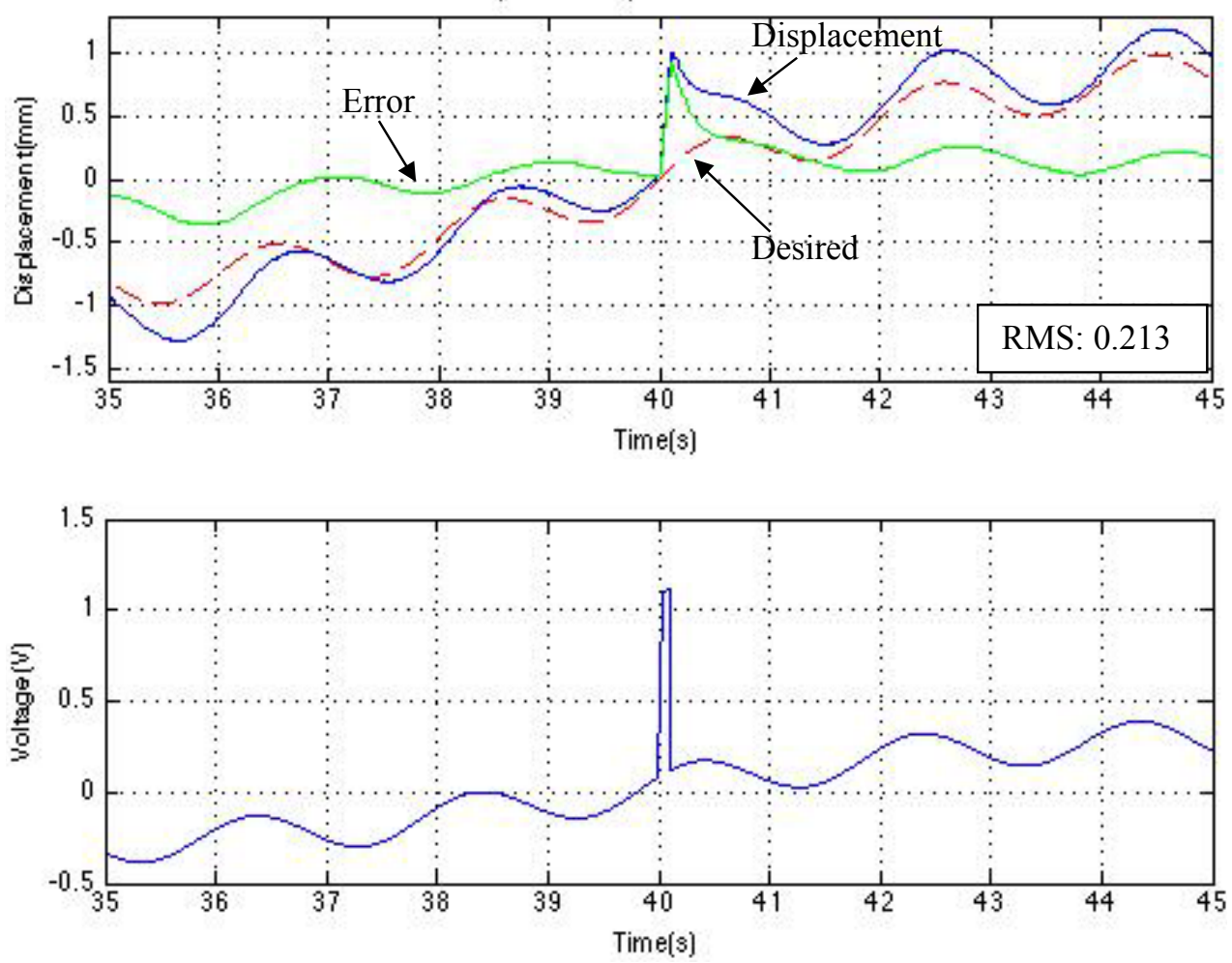

Figure 17: The experimental dynamic response with impulse disturbance under IFC.
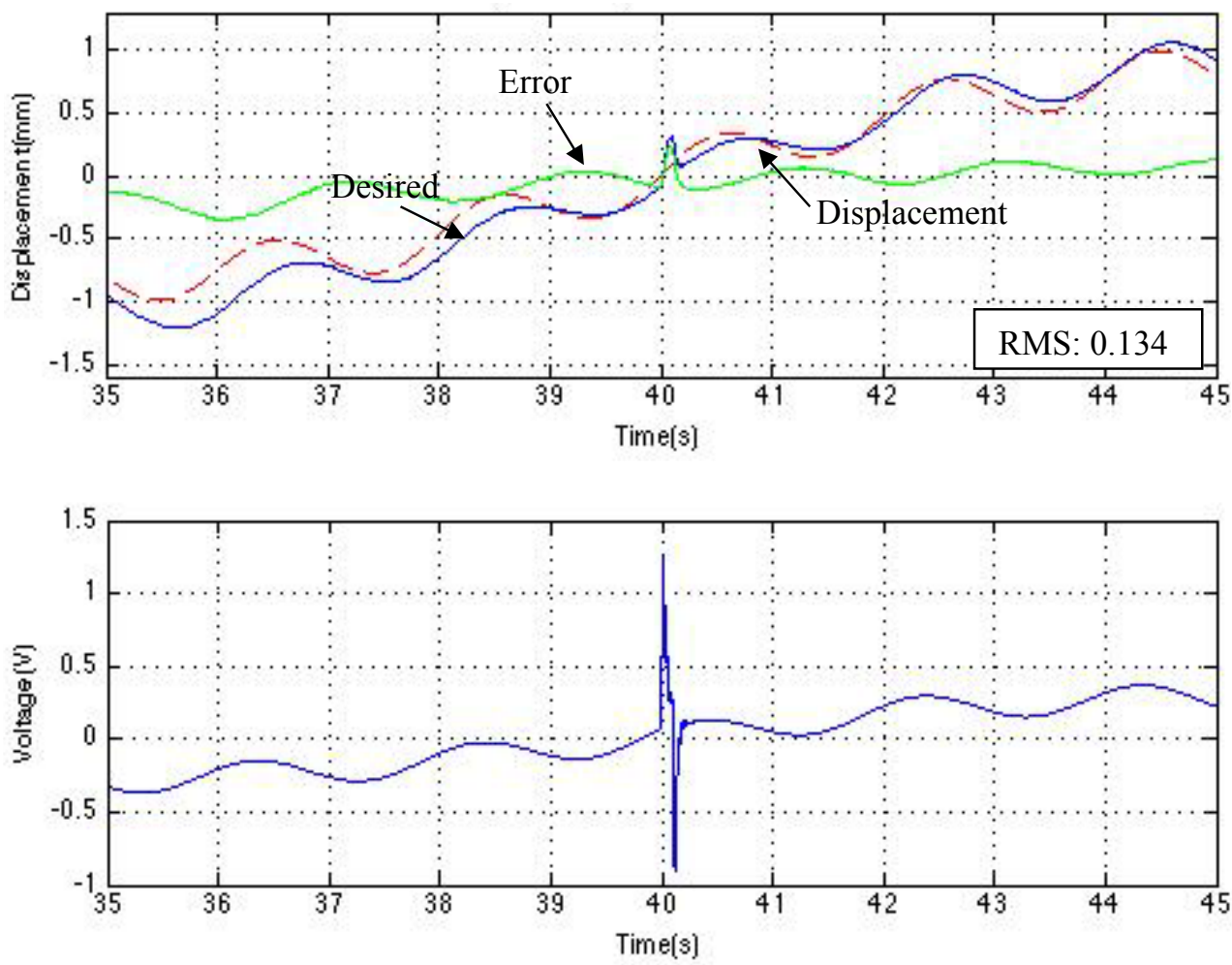

Figure 18: The experimental dynamic response with the impulse disturbance under SFC.

Table 6. The comparison of the dynamic response under IFC and SFC with impulse disturbance 


\begin{tabular}{lcll}
\hline \multicolumn{2}{l}{ Inversion-based control } & Simulated feedback control \\
\hline $\begin{array}{l}\text { Max deviation } \\
(\mathrm{mm})\end{array}$ & RMS & $\begin{array}{l}\text { Max deviation } \\
(\mathrm{mm})\end{array}$ & RMS \\
\hline 0.917 & 0.213 & 0.235 & 0.134 \\
\hline
\end{tabular}

\subsection{Dynamic Response with white noise disturbance}

As shown in figure 19 and figure 20, the displacement under SFC controller has a smaller deviation than that of IFC, and achieved the RMS of 0.171 and RMS of 0.211 , measuring a smaller overall error and better performance.

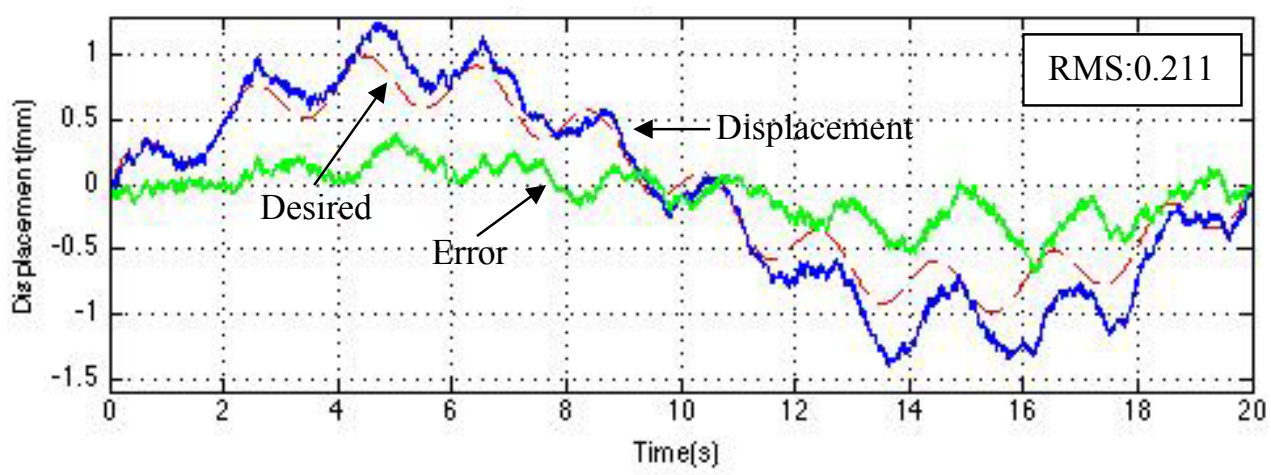

Figure 19: The experimental dynamic response with white noise disturbance under the IFC strategy.

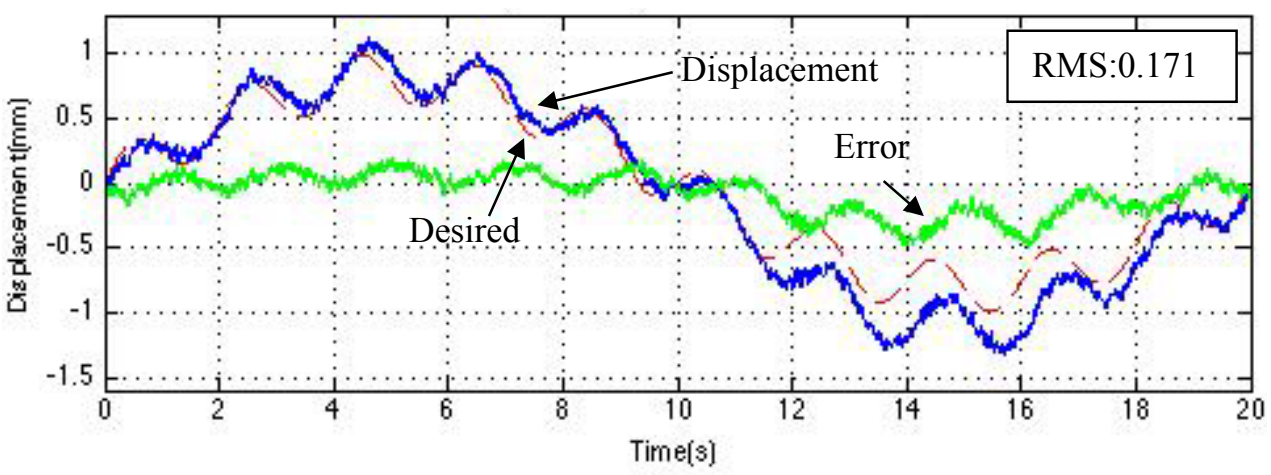

Figure 20: The Dynamic response with the white noise disturbance under the SFC strategy.

\subsection{Step Response under Extended Time}

The step displacement response of the actuator was measured over 300s. There is a trend of an increasing displacement error along with time, which is called drift. At the time of $300 \mathrm{~s}$, the displacement of the actuators is $0.573 \mathrm{~mm}$, whose error reaches $14.6 \%$ [29].

A possible explanation for this drift is the change in the electrochemical state of polymer with time [1] under a constant control input for the duration of the step. This drift also happens under the SFC which has a fixed control input calculated through the mathematical model of the actuator at the steady state. The mathematical model does not explicitly consider the drift. To address this problem effectively, we have analysed the control input calculated from the close-loop control (PID) signal at the steady-state response and found that, in order to track the input accurately, the controller output can be modified similarly to counter-balance the drift effect. As shown in figure 21 , the decrease in 
the control input signal can be fitted by a linear function. The fitted linear function and the input displacement are listed in table 7.

We define the coefficient of the $\mathrm{x}$ in the fitted linear function as slope $\mathrm{k}$. Then the relationship between slope $\mathrm{k}$ and input displacement is presented in figure 22 . We can also find the slope $\mathrm{k} 1=-$ $3.19 * 10-5$ of the linear function from the data in figure 22 when the input is $0.5 \mathrm{~mm}$. Considering this adjustment in the SFC, we can enhance the performance of the SFC controller. As shown in figure 23 and table 8 , the improvement is significant in eliminating the drift without using an external sensor.

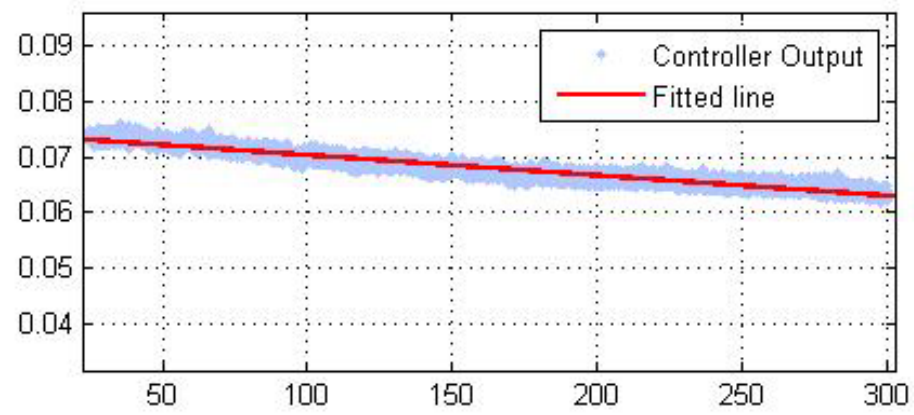

Figure 21: The PID controller output (the control signal input for the actuator) at the steady state while following the step input of $0.6 \mathrm{~mm}$. The horizontal axis is the time in seconds.

Table 7. The fitted function of the controller output signal at steady state

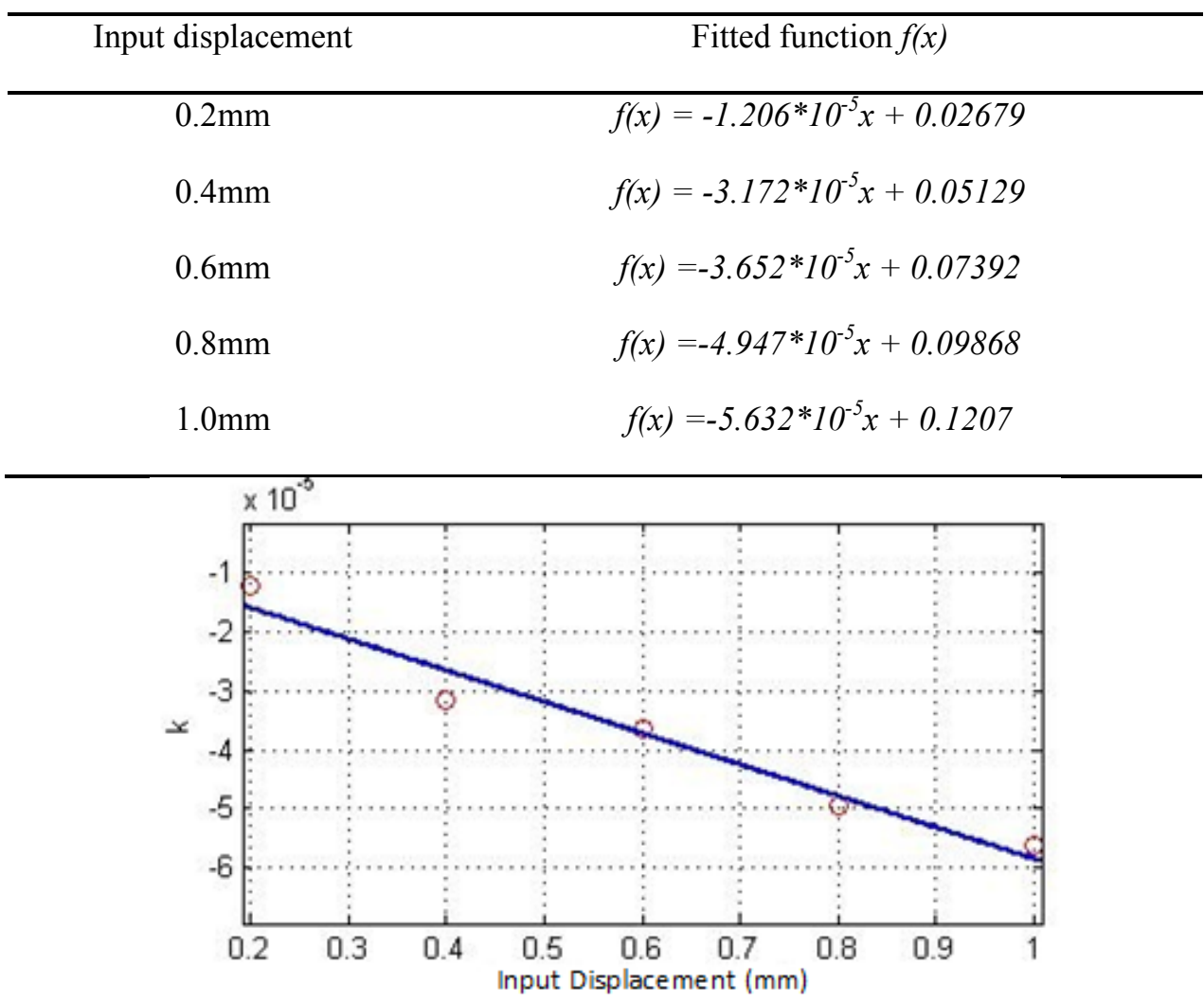

Figure 22: The relationship between the slope $k$ and the magnitude of the step displacement input. This relationship is given by $k=-5.313 * 10^{-5} * x-5.337 * 10^{-6}$. 


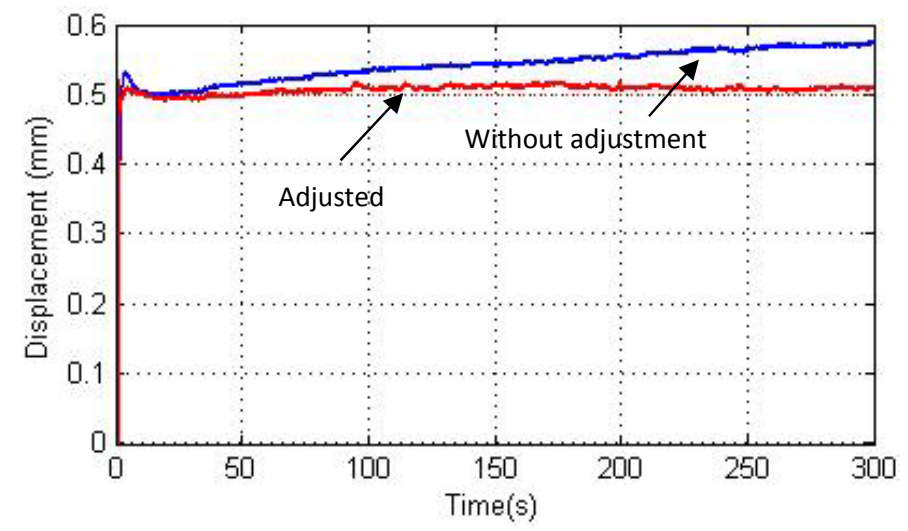

Figure 23: The comparison of the step response of the conducting polymer actuator with and without control adjustment over 300 s

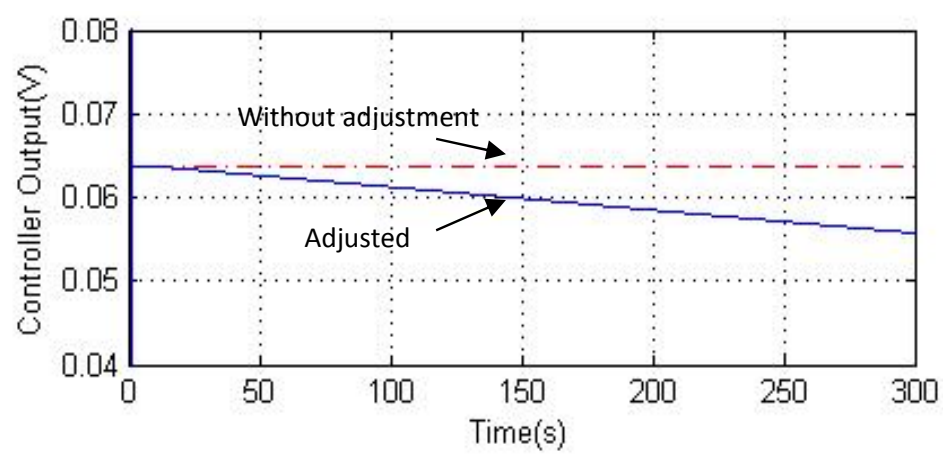

Figure 24: The comparison of the controller output with and without adjustment.

Table 8. Extended step response characteristics of CPA with and without control adjustment over 300 s.

\begin{tabular}{lclc}
\hline \multicolumn{2}{l}{ Control without adjustment } & \multicolumn{2}{l}{ Control with adjustment } \\
\hline Error at 300s & RMS & Error at 300s & RMS \\
\hline $14.6 \%(0.073)$ & 22.11 & $1.8 \%(0.009)$ & 4.55 \\
\hline
\end{tabular}

\section{Robustness analysis}

\subsection{Robustness analysis with uncertain system structure}

The actuation performance of the CPAs depends on the voltage applied between the electrodes, the thickness and the morphology of the polymer layer, the size and type of the ions, the electrolyte concentration, the environment temperature and other facts which have not been fully characterized but may affect the transport of the ions between electrode and polymer [12]. Therefore, especially the electrolyte solvent evaporation and change in electrical, mechanical and chemical properties of the actuator under a high frequency input, for example, generate significant modeling uncertainties. 
Recalling that the proposed SFC is highly dependent on the accuracy of the estimated model, it is necessary to investigate the robustness of the system with uncertain system structure and parameters.

We discuss the system robustness with uncertain structure in this section and the robustness with parameter uncertain is investigated in section 6.2.

Consider the multiplicative perturbation in figure 25 that may affect the actuator model and results in the following model [31]

$$
G_{m}(s)=G(s)[1+M(s)]
$$

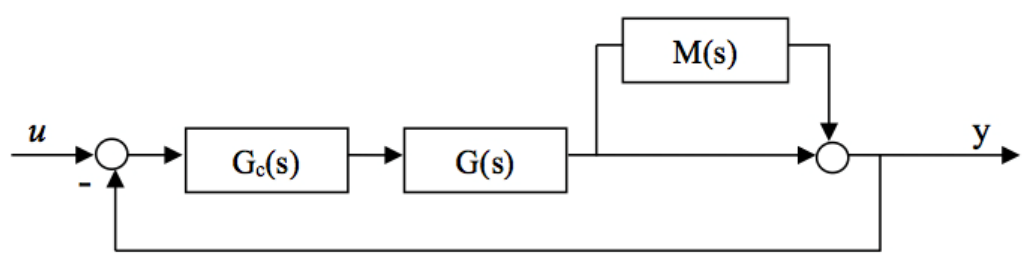

Figure 25: The schematic of the identified system with modelling uncertainty.

This form of perturbation that is bounded in magnitude is reasonable because it satisfies the properties of the model we experimentally identified. The uncertainty is small at low frequencies while it becomes larger at high frequencies, where the model is usually inaccurate.

According to the robust stability criterion, assuming that the $G_{m}(s)$ and $G(s)$ in (14) have the same number of poles in the right-hand s-plane if any [32], the system will be stable if the following condition is satisfied for all frequencies $\omega$.

$$
|M(j \omega)|<\left|1+\frac{1}{G c(j \omega) G(j \omega)}\right|
$$

For our actuator control system, the open loop transfer function is given by

$$
G_{c}(s) G(s)=\frac{1.881 s+1.9008}{s} \cdot \frac{11.72(s+2.82)}{(s+7.067)(s+1.613)}=\frac{22.05 s^{2}+84.44 s+62.82}{s^{3}+8.68 s^{2}+11.4 s}
$$

Consider the case of an unmodeled pole at $-10 \mathrm{rad} / \mathrm{s}$, which is very close to the furthest pole of the actuator at -7.067 . The multiplicative perturbation is

$$
1+M(s)=\frac{10}{s+10}
$$

The magnitude bound is then

$$
|M(j \omega)|=\left|\frac{-j \omega}{j \omega+10}\right|
$$

The $|M(j \omega)|$ and the $\left|1+1 / G_{c}(j \omega) G(j \omega)\right|$ are plotted in figure 26. It is seen that the criterion of (14) is satisfied, and the system remains stable. This shows the system can keep stable even there is bounded perturbation in the actuator model. When the unmodeled pole is further in the left-half plane, the stability margin increases. This follows that the stability of the system is not affected by the multiplicative perturbation. 


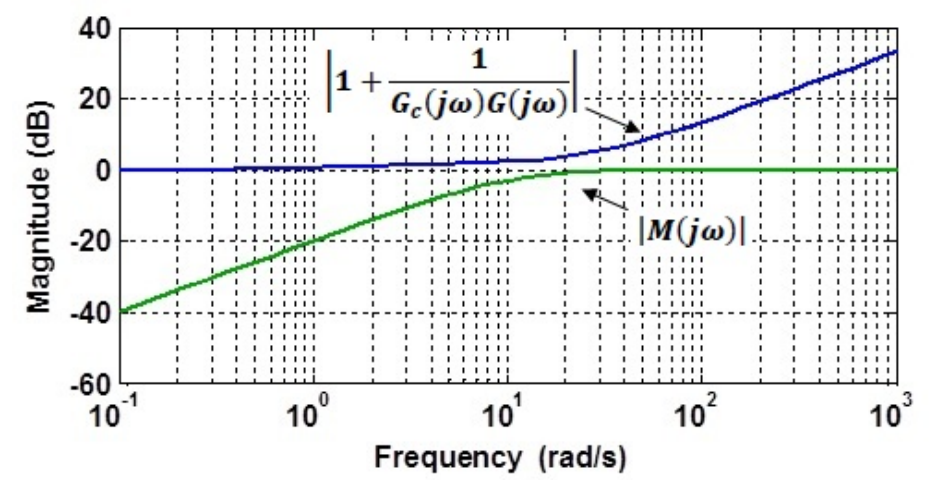

Figure 26: The robust stability criterion for CPA control system

6.2 Robustness analysis with uncertain system parameters

The actuator model $G(s)$ is in the form of

$$
G(s)=\frac{b_{1} s+b_{0}}{s^{2}+a_{1} s+a_{0}}
$$

with its coefficients within the following ranges,

$$
\begin{aligned}
& \alpha_{1} \leq a_{1} \leq \beta_{1} \\
& \alpha_{2} \leq a_{0} \leq \beta_{2}
\end{aligned}
$$

This also affects the characteristic equation. Hence the characteristic equation of the closed-loop control system with a PID controller is given by

$$
Q(s)=\left(1+b_{1} k_{d}\right) s^{3}+\left(a_{1}+b_{1} k_{p}+b_{0} k_{d}\right) s^{2}+\left(a_{0}+b_{1} k_{i}+b_{0} k_{p}\right) s+b_{0} k_{i}
$$

For each value of coefficient limits, the following four polynomials are obtained. It is proved that, the system will stable when all these four polynomial have root with negative real part [33].

$$
\begin{aligned}
& Q_{1}(s)=\left(1+b_{1} k_{d}\right) s^{3}+\left(\alpha_{1}+b_{1} k_{p}+b_{0} k_{d}\right) s^{2}+\left(\beta_{0}+b_{1} k_{i}+b_{0} k_{p}\right) s+b_{0} k_{i} \\
& Q_{2}(s)=\left(1+b_{1} k_{d}\right) s^{3}+\left(\beta_{1}+b_{1} k_{p}+b_{0} k_{d}\right) s^{2}+\left(\alpha_{0}+b_{1} k_{i}+b_{0} k_{p}\right) s+b_{0} k_{i} \\
& Q_{3}(s)=\left(1+b_{1} k_{d}\right) s^{3}+\left(\beta_{1}+b_{1} k_{p}+b_{0} k_{d}\right) s^{2}+\left(\beta_{0}+b_{1} k_{i}+b_{0} k_{p}\right) s+b_{0} k_{i} \\
& Q_{4}(s)=\left(1+b_{1} k_{d}\right) s^{3}+\left(\alpha_{1}+b_{1} k_{p}+b_{0} k_{d}\right) s^{2}+\left(\alpha_{0}+b_{1} k_{i}+b_{0} k_{p}\right) s+b_{0} k_{i}
\end{aligned}
$$

By following the Routh-Hurwitz criterion, necessary and sufficient conditions for stability are obtained as:

(1) All the coefficients of the $Q(s)$ have the same algebraic sign and

$$
\mathrm{A}\left(a_{1}+b_{1} k_{p}+b_{0} k_{d}\right)\left(a_{0}+b_{1} k_{i}+b_{0} k_{p}\right)>\left(1+b_{1} k_{d}\right) b_{0} k_{i}
$$

For the PID gains of $k_{p}=1.881, k_{i}=1.9008$ and $k_{d}=0$, the first condition is satisfied.

Consider the second condition. The values of $b_{0}$ and $b_{1}$ come from identified model. Hence the following inequality is obtained: 


$$
\begin{gathered}
\left(a_{1}+11.72 \times 1.881\right)\left(a_{0}+11.72 \times 1.9008+33.05 \times 1.881\right)>33.05 \times 1.9008 \\
a_{0} a_{1}+84.45 a_{1}+22.05 a_{0}>-1799.30
\end{gathered}
$$

(i) When $a_{0}$ is kept at its identified value of 11.4, $a_{1}>-21.39$, and still satisfying the condition (1). For $a_{0}=11.4$, the system is stable when $a_{1}$ is bigger than -21.39 . This follows that $-21.39<a_{1}<\infty$ for a stable behavior.

(ii) When $a_{1}$ is kept at its identified value of $8.68, a_{0}>-82.4$, and still satisfying the condition (1). For $a_{1}=8.68$, the system is stable when $a_{0}$ is changed from its identified value of 11.4 to -82.4 . This follows that $-82.4<a_{0}<\infty$ for a stable behavior.

The parameter ranges determined in (i) and (ii) indicate that the system is robust to the modelling uncertainties incorporated into the coefficients of the characteristic equation. These and other smart material actuators are very sensitive to the geometrical, synthesis, electrical, mechanical, and chemical parameters, and operation duration of the actuators [2, 25].The robustness of our CPA control system shows its tolerance to the model parameters uncertainty.

\section{Conclusions and further work}

This paper has proposed a new sensorless control strategy, the simulated feedback control approach, and presented its implementation on tri-layer conducting polymer actuators. This technique is able to improving the step and dynamic performance, especially under the disturbances, without the use of feedback data. However, it must be noted that the performance of the proposed control approach can deteriorate when the disturbances and their time of effect on the system are unknown.

A system transfer function model was experimentally identified and used in the simulated feedback control system in order to demonstrate its efficacy in following a displacement command and external disturbances. To obtain the best controller parameters, we have used a particle swarm optimization algorithm.

Compared with an inversion-based feedforward control strategy, the proposed simulated feedback control has shown an improved control performance including a better command-tracking ability and robustness to external disturbances.

We have proved that the SFC system can maintain its stability under unmodelled dynamics (ignoring them in the model-- multiplicative perturbation) and variations in the actuator model.

Future work involves (i) evaluating how the model parameters change with the external conditions, and (ii) considering intelligent model identification techniques in order to improve the accuracy of the actuator model and therefore, the performance of the proposed SFC approach not requiring position feedback data.

\section{Acknowledgements}

The authors would like to thank Dr. Chuc Nguyen for his help with synthesizing the conducting polymer actuators. This work was supported by the ARC Centre of Excellence for Electromaterials Science (Grant No. CE0561616). 


\section{References}

[1] G. Alici, "Guest Editorial - Introduction to the Focused Section on Electroactive Polymer Mechatronics," IEEE-ASME Transactions on Mechatronics, vol. 16, pp. 1-6, 2011.

[2] G. M. Spinks, G. Alici, S. McGovern, B. Xi, and G. G. Wallace, "Conjugated Polymer Actuators: Fundamentals," in Biomedical Applications of Electroactive Polymer Actuators, E. S. Federico Carpi, Ed., ed, 2009, pp. 195-229.

[3] K. kiyohara, T. Sugino, and K. Asaka "Molecular mechanism of ionic electroactive polymer actuators", Smart Materials and Structures, vol. 20, no.12, Nov. 2011.

[4] B.-C. Yoseph and Q. Zhang "Electroactive Polymer Actuators and Sensors", MRS Bulletin, vol. 33, no. 03, pp. 173-181, Mar. 2008

[5] G. Alici, V, Devaud, P. Renaud, and G. Spinks, "Conducting polymer microactuators operating in air," Journal of Micromechanics and Microengineering, vol. 19, p. 025017, 2009.

[6] G. Alici and D. Gunderson, "A bio-inspired robotic locomotion system based on conducting polymer actuators," presented at the IEEE/ASME International Conference on Advanced Intelligent Mechatronics, Singapore, 2009.

[7] R. Mutlu and G. Alici, "A Multi-Stable Linear Actuation Mechanism Based on Artificial Muscles", ASME Journal of Mechanical Design, Vol.132, No. 11, pp. 111001-1 -- 111001-8, November 2010.

[8] G. Alici, and N. N. Huynh, "Performance Quantification of Conducting Polymer Actuators for Real Applications: a Microgripping System", IEEE/ASME Transactions on Mechatronics, Volume 12, No.1, pp.73 -- 84, February 2007.

[9] S. W. John, G. Alici, and C. D. Cook, "Inversion-Based Feedforward Control of Polypyrrole Trilayer Bender Actuators," IEEE/ASME Transactions on Mechatronics, vol. 15, pp. 149-156, 2010.

[10] S. W. John, G. Alici, and C. D. Cook, "Towards the Position Control of Conducting Polymer Trilayer Bending Actuators with Integrated Feedback Sensor," IEEE/ASME International Conference on Advance Intelligent Mechatronics, Singapore, 2009.

[11] E. Smela, "Conjugated polymer actuators," MRS Bulletin, vol. 33, pp. 197-204, 2008.

[12] E. Smela, "Conjugated polymer actuators for biomedical applications," Advanced Materials, vol. 15, pp. 481-494, 2003.

[13] L. Bay, Torben Jacobsen, and Steen Skaarup, "Mechanism of actuation in conducting polymers: Osmotic expansion," Journal of Physical, Chemistry B, vol. 105, pp. 8492-8497, 2001.

[14] Q. Yao, G. Alici, and G. M. Spinks, "Feedback control of tri-layer polymer actuators to improve their positioning ability and speed of response," Sensors and Actuators A-Physical, vol. 144, pp. 176-184, 2008. 
[15] T. Shoa, Y. Dan Sik, K. Walus, and J. D. W. Madden, "A dynamic electromechanical model for electrochemically driven conducting polymer actuators," IEEE/ASME Trans. Mechatronics, vol. 16, no. 1, pp. 42-49, Feb. 2011

[16] A. D. Price, and H. E. Naguib, "A unified multiphysics finite element model of the polypyrrole trilayer actuation mechanism", Journal of Intelligent Material Systems and Structures, vol. 24, no. 5, pp. 548-558, 2013.

[17] C. Druitt, G. Alici, "Intelligent Control of Electroactive Polymer Actuators Based on Fuzzy and Neuro-Fuzzy Methodologies", IEEE/ASME Transactions on Mechatronics, October 2013 (under review; Minor revision).

[18] F. Yang, X. Tan, and G. Alici, "Robust Adaptive Control of Conjugated Polymer Actuators," IEEE Transactions on Control System Technology, vol. 16, pp. 600-612, 2008.

[19] X. Wang, G. Alici, and C. H. Nguyen, "Adaptive sliding mode control of tri-layer conjugated polymer actuators," Smart Materials and Structures, vol. 22, pp. 1-8, 2013.

[20] C. Tang and G. Alici, "Evaluation of length-scale effects for mechanical behaviour of microand nanocantilevers: I. Experimental determination of length-scale factors," Journal of Physics D: Applied Physics, vol. 44, pp. 1-12, 2011.

[21] G. Alici, N. N. Huynh, "Predicting force output of trilayer polymer actuators," Sensors and Actuators A, vol. 132, pp. 616-625, 2006.

[22] S. W. John, G. Alici, and C. D. Cook, "Validation of a Resonant Frequency Model for Polypyrrole Trilayer Actuators", IEEE/ASME Transactions on Mechatronics, Vol.13, No.4, pp.401 -- 409, August 2008.

[23] G. Alici, "An effective modelling approach to estimate nonlinear bending behaviour of cantilever type conducting polymer actuators," Sensors \& Actuators: B. Chemical, vol. 141, pp. 284-292, 2009.

[24] Y. Fang, X. Tan, and G. Alici, "Redox Level-Dependent Impedance Model for Conjugated Polymer Actuators", Sensors and Actuators: B. Chemical, Vol. 132, No.1, pp. 182 - 190, May 2008.

[25] C. H. Nguyen, G. Alici, and G. G. Wallace, "Modelling trilayer conjugated polymer actuators for their sensorless position control," Sensors and Actuators A-Physical, vol. 185, pp. 82-91, 2012.

[26] J. Kennedy and R. Eberhart, "Particle swarm optimization " in IEEE International Conference on Neural Networks, Perth, WA, 1995, pp. 1942-1948.

[27] R. Poli, J. Kennedy, and T. Blackwell, "Particle swarm optimization: An overview," Swarm Intelligence, vol. 1, pp. 33-57, 2007.

[28] Z.-L. Gaing, "A particle swarm optimization approach for optimum design of PID controller in AVR system," IEEE Transactions on Energy Conversion, vol. 19, pp. 384-391, 2004. 
[29] X. Xiang, G. Alici, W. Li, "Control of Conducting Polymer Actuators without feedback: Simulated Feedback Control Approach”, 2013 IEEE/ASME International Conference on Advanced Intelligent Mechatronics, pp.900 -- 905, Wollongong, Australia, July 2013.

[30] C.-C. Kao, C.-W. Chuang, and R.-F. Fung, "The self-tuning PID control in a slider-crank mechanism system by applying particle swarm optimization approach," Mechatronics, vol. 16, pp. 513-522, 2006.

[31] J. C. Doyle, A. B. Francis, and A. R. Tannenbaum, Feedback Control Theory, Macmillan, New York, 1992.

[32] R. C. Dorf and R. H. Bishop, Eds., Modern Control Systems. Pearson Education, Inc., 2008.

[33] W. J. Grantham and T. L. Vincent, Modern Control Systems Analysis and Design, John Wiley \& Sons, New York, 1993. 